\title{
Dialysierte Chlorella, ein neues Versuchsmaterial zur Untersuchung der Photosynthese
}

\author{
Otto Warburg, Günter Krippahl und Arnold Lehmann \\ Max-Planck-Institut für Zellphysiologie, Berlin-Dahlem \\ (Z. Naturforschg. 23 b, 1076-1079 [1968]; eingegangen am 4. Mai 1968)
}

\begin{abstract}
Als neues Versuchsmaterial der Photosynthese wird dialysierte Chlorella beschrieben. Mit Hilfe der dialysierten Chlorella wurden zwei bisher unbekannte stöchiometrische Lichtreaktionen entdeckt, die Abspaltung von Kohlensäure aus der aeroben Kohlensäure, sowie die Umwandlung der aeroben Kohlensäuren in den Photolyten.
\end{abstract}

Wir berichten in dieser Arbeit über ein neues Versuchsmaterial, die „dialysierte Chlorella“, mit der es gelingt, Chlorella vollständiger von dem aktiven Mangan zu befreien, als es mit den früheren Methoden möglich gewesen ist. Wir berichten ferner über zwei bisher unbekannte stöchiometrische Lichtreaktionen, die wir mit Hilfe der dialysierten Chlorella entdeckt haben: über die Umwandlung der aeroben Kohlensäure in den Photolyten durch Licht sehr niedriger Intensität und über die Abspaltung der aeroben Kohlensäure durch Licht höherer Intensität, wobei Kohlensäure im Licht erscheint. Die Quantenausbeute dieser photochemischen Kohlensäureentwicklung betrug in den bisherigen Versuchen nahezu

$$
\varphi=0,5\left[\frac{\mu \mathrm{Mol} \mathrm{CO}_{2}}{\mu \mathrm{Mol} \text { abs. Lichtquanten }}\right] .
$$

Da Chlorella mit Hilfe von Sauerstoff etwa 1/20 ihres Zellvolumens an aerober Kohlensäure binden und in den Photolyten umwandeln kann ${ }^{1}$, so liegen hier zur Zellsubstanz stöchiometrische Reaktionen der Kohlensäure vor, die wegen der Größe ihres Umsatzes genau gemessen werden können.

\section{Dialyse der Chlorella}

Wir dialysieren Chlorella, die in $m / 50-\mathrm{KH}_{2} \mathrm{PO}_{4}$ suspendiert ist, bei $+3{ }^{\circ} \mathrm{C}$ in bewegten Zellophanschläuchen, an denen in $24 \mathrm{Stdn} .50 \mathrm{~lm} / 50-\mathrm{KH}_{2} \mathrm{PO}_{4}$ vorbeifließen.

Auf diese Weise dialysierte Chlorella ist nicht wesentlich geschädigt, wovon man sich durch Übertragung in Kulturlösung, der die Mikroelemente zugesetzt sind, überzeugen kann. Andererseits ist die dialysierte, in $m / 50-\mathrm{KH}_{2} \mathrm{PO}_{4}$ suspendierte Chlorella

1 O. Warburg u. G. Krippahl, Biochem. Z. 346, 418 [1967]; 346, 429 [1967] ; Z. Naturforschg. 15 b, 788 [1960]. nicht imstande, im Dunkeln Photolyt zu bilden, obwohl ihre Fähigkeit zur Bildung aerober $\mathrm{CO}_{2}$ erhalten ist. Mit Hilfe der Dialyse der Chlorella kann man also Chlorella gewinnen, in der die Umwandlung der aeroben $\mathrm{CO}_{2}$ in Photolyten vollständig gehemmt ist. Zusatz von sehr wenig Mangansulfat zu der dialysierten Chlorella stellt die Fähigkeit zur Photolytbildung im Dunkeln sofort wieder her. Andere Mikroelemente, einzeln oder gemeinsam, waren wirkungslos, wenn das Mangan fehlte. Wirkungslos war auch Magnesiumsulfat in großen Mengen. $140 \mu \mathrm{Mol}$ Magnesiumsulfat, zu $100 \mathrm{~mm}^{3}$ Chlorella zugesetzt, konnten $0,1 \mu \mathrm{Mol}$ Mangansulfat bei der Photolytbildung nicht ersetzen.

Die 24-stdg. Dialyse der Chlorella extrahiert weder die löslichen Fermente noch die Aminosäuren der Chlorella, so daß die dialysierte Chlorella als Versuchsobjekt komplementär zu den 1944 eingeführten ${ }^{2}$ mit Wasser gewaschenen Spinatgrana ist. Von beschallter Chlorella unterscheidet sich dialysierte Chlorella als Versuchsobjekt dadurch, daß sie $\mathrm{CO}_{2}$ fixiert, während beschallte Chlorella die im Licht reduzierte $\mathrm{CO}_{2}$ immer quantitativ zu $\mathrm{CO}_{2}$ zurückoxydiert.

\section{Spaltung der aeroben Kohlensäure durch Licht}

Sättigt man dialysierte Chlorella 30 Dunkelminuten mit $10 \mathrm{Vol} . \% \mathrm{CO}_{2}$-Luft, so wird trotz des Manganmangels die aerobe $\mathrm{CO}_{2}$ gebildet, aber nichts davon wird in den Photolyten umgewandelt. Der Beweis ist, daß beim Übergang von dunkel zu hell zunächst kein $\mathrm{O}_{2}$ erscheint.

Statt des $\mathrm{O}_{2}$ jedoch erscheint beim Übergang von dunkel zu hell $\mathrm{CO}_{2}$, deren Endwert in dem Versuch der Abb. 1 in 2 Hellminuten erreicht ist. Die

2 O. Warburg u. W. Lürtgens, Naturwissenschaften 32, 161, 301 [1944]. 
aerobe $\mathrm{CO}_{2}$, die im Verlauf des Versuchs durch Einkippen von Fluorid gemessen wurde, nahm dabei in dem $\mathrm{Ma} \beta \mathrm{ab}$, als die entwickelte $\mathrm{CO}_{2}$ zunahm, so daß also die $\mathrm{CO}_{2}$, die das Licht entwickelt, aus der aeroben $\mathrm{CO}_{2}$ entwickelt wird.

Im ganzen war das Ergebnis derartiger Versuche, daß beim Übergang von dunkel zu hell aus der aeroben $\mathrm{CO}_{2}$ nur $\mathrm{CO}_{2}$ und aus dem Photolyten nur $\mathrm{O}_{2}$ entwickelt wird; daß also aus einem Gemisch von aerober $\mathrm{CO}_{2}$ und Photolyt ein Gemisch von $\mathrm{CO}_{2}$ und $\mathrm{O}_{2}$ entwickelt wird. Auch aus nichtdialysierten Zellen, deren Mangangehalt normal ist, kann man Gemische von $\mathrm{CO}_{2}$ und $\mathrm{O}_{2}$ entwickeln, wenn man $\mathrm{zu}$ früh von dunkel zu hell übergeht. Zum Beispiel ist nach 10 Dunkelminuten die Hauptmenge der aeroben $\mathrm{CO}_{2}$ bereits gebildet, aber die Umwandlung in den Photolyten ist noch nicht vollständig, so daß nach zu kurzer Dunkelheit Licht ein Gemisch von $\mathrm{CO}_{2}$ und $\mathrm{O}_{2}$ entwickelt.

\section{Umwandlung der aeroben Kohlensäure in den Photolyten durch Licht}

Wenn man die aerobe $\mathrm{CO}_{2}$ in den dialysierten manganarmen Zellen durch Licht vollständig zersetzt hat und dann weiter belichtet, so beginnt alsbald eine Aufnahme von $\mathrm{CO}_{2}$ und eine Entwicklung von $\mathrm{O}_{2}$, woraus hervorgeht, daß nunmehr im Licht Photolyt gebildet wird; während doch vorher, im Dunkeln, kein Photolyt gebildet worden war. Manganarme Zellen, die wegen ihrer Manganarmut im Dunkeln keinen Photolyt bilden können, sind also im Licht imstande, Photolyt zu bilden. Die graphische Darstellung eines derartigen Versuchs in Abb. 1 zeigt, wie nach 2 Hellminuten die Photolytbildung bereits beginnt.

Zur näheren Untersuchung der Erscheinung war es notwendig, Bildung und Spaltung des Photolyten zeitlich von einander zu trennen. Dies gelang, weil zur Bildung des Photolyten im Licht viel kleinere Lichtintensitäten genügen, als zur Spaltung des Photolyten. Abb. 2 zeigt, daß die aerobe $\mathrm{CO}_{2}$ vollständig in Photolyt umgewandelt wird, wenn man in dialysierte manganarme Zellen 60 Min. eine Lichtintensität $J=1,5 \mathrm{~mm}^{3}$ Quanten pro Min. einstrahlt; während Abb. 1 zeigt, daß wir $50 \mathrm{~mm}^{3}$ Quanten pro Min. einstrahlen, um den Photolyten zu spalten.

Bei beiden Lichtreaktionen, der Bildung des Photolyten und der Spaltung des Photolyten, haben wir bisher keinen spezifischen Einfluß der Wellen-

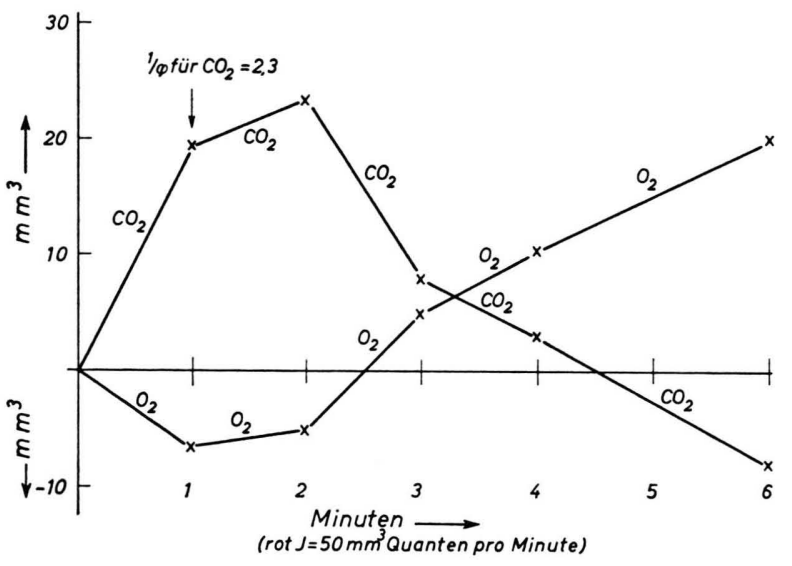

Abb. 1. Chlorella, suspendiert in $m / 50-\mathrm{KH}_{2} \mathrm{PO}_{4}$, wurde bei $+3{ }^{\circ} \mathrm{C} 24$ Stdn. dialysiert. Dann wurde bei $10{ }^{\circ} \mathrm{C}$ der Stoffwechsel mit dem Gefäßpaar ungleichen Volumens gemessen. Das Volumen von Gefäß I war $17,86 \mathrm{~cm}^{3}$, das Volumen von Gefäß II 33,69 $\mathrm{cm}^{3}$, das Volumen der eingefüllten Zellsuspension $7,2 \mathrm{~cm}^{3} \mathrm{~m} / 50-\mathrm{KH}_{2} \mathrm{PO}_{4}$, die je $100 \mathrm{~mm}^{3}$ Chlorella enthielt, deren Chlorophyllgehalt etwa 1,2 Mikromol betrug. Der Gasraum enthielt 10 Vol.-\% $\mathrm{CO}_{2}$-Luft. Aus diesen Daten ergibt sich die Berechnung von $x_{2}$ und $x_{\mathrm{CO}_{2}}$ aus den beobachteten Druckänderungen $H^{\prime}$ und $H$

$$
\begin{aligned}
x_{\mathrm{O}_{2}} & =H^{\prime} \times 4,035-H \times 7,30\left[\mathrm{~mm}^{3}\right], \\
x \mathrm{CO}_{2} & =-H^{\prime} \times 5,33+H \times 13,05\left[\mathrm{~mm}^{3}\right] .
\end{aligned}
$$

Die Gefäße wurden zunächst 30 Dunkelminuten im Thermostaten zwecks Sättigung mit aerober $\mathrm{CO}_{2}$ geschüttelt. Dann wurden $50 \mathrm{~mm}^{3}$ Quanten rotes Licht pro Min. eingestrahlt, die zu 90\% absorbiert wurden. $t_{0}$ in der Abb. 1 ist die Zeit, zu der die Belichtung begann. Folgende Ablesungen wurden für die beiden ersten Hellminuten nach der Verdunkelung erhalten:

$$
\begin{aligned}
& \text { Gefäß I } \\
& \text { Gefäß II } \\
& H^{\prime} \text { [mm Brodie] } \quad H \text { [mm Brodie] } \\
& \begin{array}{lll}
5^{\prime} \text { dunkel } & -1 & -0,5
\end{array} \\
& l^{\prime} \mathrm{d}, \mathrm{l}^{\prime} \operatorname{rot}(J=50) \quad \mathrm{l}^{\prime} \mathrm{d} \quad+3,0 \quad+2,0 \\
& 2,5^{\prime} \mathrm{d}-1,5 \quad-1,5 \\
& 2,5^{\prime} \mathrm{d}-1,5 \quad-1,0 \\
& 20^{\prime} \mathrm{d}-4,5 \quad-2,5 \\
& 5^{\prime} \mathrm{d}-1,5 \quad-0,5
\end{aligned}
$$

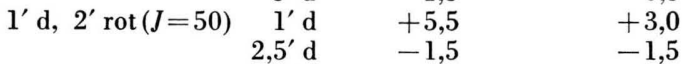

$$
\begin{aligned}
& 2,5^{\prime} \mathrm{d} \quad-1,5 \quad-1,0
\end{aligned}
$$

Die Abb. 1 zeigt, daß in den beiden ersten Hellminuten kein $\mathrm{O}_{2}$, sondern nur Kohlensäure entwickelt wird; daß aber bei weiterer Belichtung in einigen Hellminuten die normale Photosynthese entsteht, d. h. $\mathrm{CO}_{2}$-Absorption und $\mathrm{O}_{2}$-Entwicklung ${ }^{5}$.

längen gefunden. - Während die Bildung des Photolyten im Dunkeln eine Mangankatalyse ist, konnten wir bisher nicht entscheiden, ob auch die Bildung des Photolyten im Licht Mangan benötigt, oder nur weniger Mangan benötigt, als die Bildung

5 O. Warburg u. G. Krippahl, Biochem. Z. 346, 428 [1967]. 


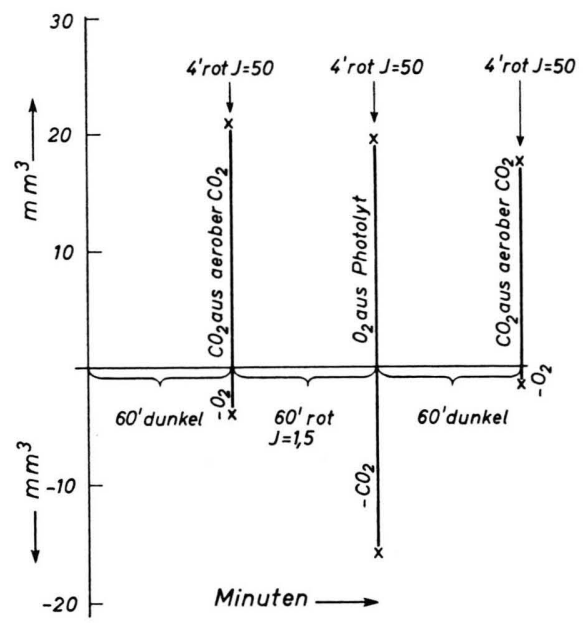

$$
\begin{gathered}
\text { Gefäß I } \\
H^{\prime}[\mathrm{mm}] \\
60^{\prime} \mathrm{d} \\
5^{\prime} \mathrm{d} \\
1^{\prime} \mathrm{d} \\
2,5^{\prime} \mathrm{d} \\
2,5^{\prime} \mathrm{d} \\
55^{\prime} \operatorname{rot}(J=1,) \\
5^{\prime} \operatorname{rot}(J=1,5) \\
\mathrm{l}^{\prime} \mathrm{d}, 4^{\prime} \operatorname{rot}(J=50) \\
\\
\mathrm{l}^{\prime} \operatorname{rot}(J=1,5) \\
2,5^{\prime} \operatorname{rot}(J=1,5) \\
2,5^{\prime} \operatorname{rot}(J=1,5) \\
55^{\prime} \mathrm{d} \\
5^{\prime} \mathrm{d} \\
1^{\prime} \mathrm{d} \\
2,5^{\prime} \mathrm{d} \\
\mathrm{l}^{\prime} \mathrm{d}, 4^{\prime} \operatorname{rot}(J=50) \\
2,5^{\prime} \mathrm{d}
\end{gathered}
$$

Das Ergebnis, das in der Abb. 2 graphisch dargestellt ist, zeigt, daß in 60 Dunkelminuten kein Photolyt gebildet wurde, sondern nur aerobe Kohlensäure; da $\beta$ in den folgenden 60 Min., in denen $J=1,5 \mathrm{~mm}^{3}$ Quanten pro Min. eingestrahlt wurden, die normale Menge Photolyt gebildet wurde, so daß der

des Photolyten im Dunkeln. Auch die dialysierten manganarmen Zellen enthielten inner noch meßbare Mengen Mangan in $100 \mathrm{~mm}^{3}$ Chlorella.

Die Tatsache, daß Licht den Photolyten nicht nur spaltet, sondern auch bildet, beseitigt einen Widerspruch $^{3}$, der seit der Entdeckung des Photolyten existierte. $100 \mathrm{~mm}^{3}$ normale Chlorella können im Dunkeln in 30 Min. nur etwa $25 \mathrm{~mm}^{3}$ Photolyt bilden, während die gleiche Menge Chlorella bei Sättigung mit Licht in der gleichen Zeit etwa $1500 \mathrm{~mm}^{3}$ $\mathrm{O}_{2}$ entwickeln, also $1500 \mathrm{~mm}^{3}$ Photolyt bilden kann. Die Maximalgeschwindigkeit der Photolytbildung im

3 Allgemeines über Luminofermente vgl. O. Warburg, G. KrIPPAHL u. andere, Z. Naturforschg. 9 b, 769 [1954]; Hoppe-Seylers Z. physiol. Chem. 332, 225 [1963], besonders S. 233.
Abb. 2. Chlorella, suspendiert in $m / 50-\mathrm{KH}_{2} \mathrm{PO}_{4}$, wurde bei $+3{ }^{\circ} \mathrm{C} 40$ Stdn. dialysiert. Dann wurde der Stoffwechsel mit dem Gefäßpaar ungleichen Volumens gemessen. Die Gefäße waren die gleichen, wie im Versuch der Abb. 1. Die gleiche Chlorella-Menge $\left(100 \mathrm{~mm}^{3}=\sim 1,2 \mu \mathrm{Mol}\right.$ Chloroplyll Zellen) suspendiert in $7,2 \mathrm{~cm}^{3} \mathrm{~m} / 50-\mathrm{KH}_{2} \mathrm{PO}_{4}$ wurde in jedes Gefäß eingefüllt. Anders als im Versuch der Abb.l enthielt der Gasraum 20 Vol. $\% \mathrm{CO}_{2}$-Luft und der Stoffwechsel wurde bei $20^{\circ} \mathrm{C}$ gemessen. Dann waren die Formeln zur Berechnung von $x_{0_{2}}$ und $x_{\mathrm{CO}_{2}}$ aus den beobachteten Druckänderungen $H^{\prime}$ und $H$

$$
\begin{aligned}
x_{0_{2}} & =H^{\prime} \times 4,36-H \times 8,36\left[\mathrm{~mm}^{3}\right], \\
x \mathrm{CO}_{2} & =-H^{\prime} \times 5,45+H \times 13,5\left[\mathrm{~mm}^{3}\right] .
\end{aligned}
$$

Das Wesentliche des Versuchs war die verschiedene Vorbehandlung der Zellen, ehe mit Licht die aerobe $\mathrm{CO}_{2}$ oder der Photolyt gespalten wurden, wobei zur Spaltung $4.50 \mathrm{~mm}^{3}$ Quanten roten Lichts in 4 Min. eingestrahlt wurden. Die Vorbehandlung betrug immer eine Stde. und geschah entweder in Dunkelheit oder bei Einstrahlung von rotem Licht sehr niedriger Intensität. Wir fanden :

$$
\begin{aligned}
& \text { Gefäß II Ergebnis } \\
& H[\mathrm{~mm}] \\
& -8 \\
& \left.\begin{array}{l}
-0,5 \\
+3,0
\end{array}\right\}+5,1 \quad x_{0_{2}}-4 \\
& \left.\begin{array}{l}
+3,0 \\
-1,0
\end{array}\right\}+5,1 \quad \begin{array}{l}
x_{2}-4 \\
x \mathrm{CO}_{2}+21
\end{array} \\
& -0,5 \\
& -5,0 \\
& \left.\begin{array}{l}
-0,5 \\
+1,5
\end{array}\right\}+2,6 \quad x_{02}+19,5 \\
& \left.\frac{+1,5}{-0,5}\right\}^{+2,6} \quad x_{\mathrm{CO}_{2}}-16,1 \\
& -0,5 \\
& -5,5 \\
& \left.\begin{array}{l}
-0,5 \\
+3,0
\end{array}\right\}+5,1 \quad x_{0_{2}}-1,4 \\
& \begin{array}{ll}
+3,0 \\
-1,0
\end{array} \int^{+5,1} \begin{array}{ll}
x_{\mathrm{CO}_{2}}+1,4 \\
\end{array} \\
& -0,5
\end{aligned}
$$

4-Min.-Test mit $J=50$ normale Photosynthese ergab; und daß in den darauf folgenden Dunkelminuten kein Photolyt gebildet wurde, so daß der Test mit $J=50$ nur $\mathrm{CO}_{2}$, keinen Sauerstoff entwickelte.

Licht betrug also das 60-fache der Photolytbildung im Dunkeln. Durch die Entdeckung, daß Licht Photolyt nicht nur spaltet, sondern auch bildet, ist dieser Widerspruch nunmehr beseitigt.

\section{Phenanthrolin ${ }^{4}$}

Gleichgültig, ob Licht in intakter Chlorella oder in beschallter Chlorella oder in gewaschenen Spinatgrana $\mathrm{O}_{2}$ entwickelt; gleichgültig, ob das Oxydans der energieliefernden Rückreaktion molekularer $\mathrm{O}_{2}$ oder Ferricyankalium oder Benzochinon oder T.P.N.

\footnotetext{
4 O. Warburg u. G. Krippahl, Ortho- und meta-Phenanthrolin in Chlorella. Weiterentwicklung der zellphyiologischen Methoden. Georg Thieme, Stuttgart u. Interscience, New York
} 1962. 
ist, immer wird die $\mathrm{O}_{2}$-Entwicklung im Licht durch $n / 1000$-ortho-Phenanthrolin vollständig und reversibel gehemmt. So schien es bisher, daß die Phenanthrolinhemmung spezifisch für die $\mathrm{O}_{2}$-Entwicklung im Licht sei.

Diese Auffassung muß nunmehr aufgegeben werden. Denn wir fanden, daß auch die $\mathrm{CO}_{2}$-Entwicklung aus aerober $\mathrm{CO}_{2}$ im Licht durch $n / 1000$-Phenanthrolin vollständig und reversibel gehemmt wird; und $\mathrm{da} \beta$ auch die Umwandlung der aeroben $\mathrm{CO}_{2}$ in den Photolyten durch $n / 1000$-Phenanthrolin vollständig gehemmt wird. Das gemeinsame aller dieser durch $n / 1000$-Phenanthrolin hemmbaren Lichtreaktionen ist eine katalytische Wirkung des belichteten Chlorophylls.

$\mathrm{Da}$ aber diese Katalysen durch viel weniger Phenanthrolin gehemmt werden, als sie Chlorophyll enthalten, so kann der Phenanthrolinhemmung keine Reaktion zwischen Chlorophyll und Phenanthrolin zugrundeliegen, sondern der Reaktionspartner des Phenanthrolins muß eine Substanz sein, deren Menge klein ist gegen die Chlorophyllmenge, die inaktiviert wird. Diese Substanz könnte Mangan sein, dessen Komplex mit Phenanthrolin nach Versuchen von Krippahl, Birkicht und Stevens eine Bande im UV bei $344 \mathrm{~m} \mu$ hat, deren $\beta$-Wert $0,96 \mathrm{~cm}^{2} / \mu \mathrm{Mol}$ beträgt, wenn der $\mathrm{pH}$ Wert 6 und das Phenanthrolin in 10 - bis 40 -fachem Überschuß ist. Doch liegt ein Beweis zur Zeit noch nicht vor, obwohl wir nunmehr e ine Mangankatalyse in Chlorella kennen, die durch Phenanthrolin antikatalytisch gehemmt wird, nämlich die Umwandlung der aeroben $\mathrm{CO}_{2}$ in den Photolyten im Dunkeln *.

\footnotetext{
* Zum Nachweis dieser Antikatalyse des Phenanthrolins war eine Methode notwendig, um das Phenanthrolin schnell aus den gehemmten Zellen zu entfernen. Wir benutzten dazu Zinksulfat, das wir aus einer Birne in den Hauptraum eines Manometriegenfäßes einkippten, in dem sich die Chlorella und das hemmende Phenanthrolin befanden. In wenigen Min. wird dann das Phenanthrolin als komplexes Zinksalz gebunden.
} 